\title{
DESIGN AND FABRICATION OF A TUNABLE INP-BASED VCSEL USING A ELECTRO-OPTIC INDEX MODULATOR
}

\author{
C. Levallois ${ }^{1}$, S. Richard ${ }^{1}$, A. Le Corre ${ }^{1}$, S. Loualiche ${ }^{1}$, \\ B. Caillaud ${ }^{2}$, J-L. de Bougrenet de la Tocnaye ${ }^{2}$, L. Dupont ${ }^{2}$. \\ ${ }^{1}$ Laboratoire d'Étude des Nanostructures à Semiconducteurs, UMR-CNRS FOTON nº 6082 \\ INSA Rennes, 20 av. des Buttes de Coësmes, 35043 Rennes Cedex, France. \\ ${ }^{2}$ Optics department, UMR-CNRS FOTON n ${ }^{\circ} 6082$ \\ ENST Bretagne, CS 83818, 29238 Brest Cedex, France
}

\begin{abstract}
We present the first vertical surface emitting laser (VCSEL) operating at 1.55- $\mu \mathrm{m}$ comprising a electro-optic modulator inside its cavity. This material consists of nematic liquid crystal dispersed in a polymer material (nano-PDLC). This first VCSEL exhibits a $10 \mathrm{~nm}$ tuning range and an excellent side-mode suppression ratio higher than $20 \mathrm{~dB}$ over the whole spectral range. The device is formed by a conventional InP-based active region with an epitaxial and a dielectric Bragg mirror. The nano-PDLC layer length, close to $6 \mu \mathrm{m}$, is in agreement with a tunable laser emission without mode-hopping. Another decisive advantage, compared to mechanical solutions, is the tuning response time which is close to a few $10 \mu$ s to scan the full spectral range, making this device appropriate for some access network functions. This first version is optically pumped and requires 170 volts to obtain a $10 \mathrm{~nm}$ tunability.

\section{Introduction}

Long-wavelength vertical cavity surface emitting lasers (VCSEL) operating at $1.3 \mu \mathrm{m}$ and $1.5 \mu \mathrm{m}$ have been extensively studied during the last decade. Their circular and spatial single-mode beam provide very efficient fiber coupling. They also offer other advantages like wafer testing before packaging or fabrication in array configuration, well suited for low cost front end equipments of future Passive Optical Networks (PON). In order to increase communication capacities of the embedded fibers, advanced VCSELs with a tunable emission have been investigated (1). These kinds of devices make them suitable for wavelength division multiplexing (WDM) applications in metro and local access networks (MAN and LAN). One of the most investigated device is the tunable VCSEL based on micro-electromechanical systems (MEMS). Electrically or optically

suffers from polarization dependence due to the birefringence of the nematic LC.

Many works demonstrated the advantages of nanopolymer dispersed liquid crystal (nano-PDLC) compared with bulk LC or usual PDLC (6)(7). This material is polarization insensitive and provides fast tuning speed which are interesting properties for a VCSEL. Thus, in this paper, we report the first tunable VCSEL operating in the C-band based on such a material. The device is easy to fabricate and robust. The VCSEL comprises a conventional InP based active region and an InP/InGaAsP bottom Bragg reflector. This half-cavity is combined with a $\mathrm{SiO}_{2} / \mathrm{TiO}_{2}$ front dielectric Bragg reflector and the electro-optic phase modulator in order to tune the laser emission wavelength. This optically pumped VCSEL requires less than $170 \mathrm{~V}$ to achieve a $10 \mathrm{~nm}$ tunability and presents a laser emission at room-temperature (RT) under pulsedoperation.
\end{abstract} pumped MEMS-VCSEL offer an ideal solution for mode-hop free and wide wavelength tuning (2)(3). However, this solution requires a complex processing and is limited by their fragility. Furthermore, MEMS-VCSEL exhibit a relatively low switching speed which depends of the MEMS structure size (few ms) (4). An other way to obtain a VCSEL with a wide tuning range is to use a liquid crystal as an electro-optic material placed inside the cavity (5). The liquid crystal (LC) solution allows to obtain a large refractive index variation, but the tuning speed is no faster than a millisecond and it also

\section{Nano-PDLC description}

The tunable part of the device consists of a suspension of LC droplets in a host medium. The LC directors axes within a droplet are determined by the LC interaction at the droplet boundary and it is therefore vary nearly random from droplet to droplet in the absence of an external electric field $E$. As shown in Fig. 1, with an applied electric field along $z, \mathrm{LC}$ directors are aligned with $E$, causing index increase along $z$, 
whereas $n_{x}$ and $n_{y}$ decrease. For a propagation vector $k$ parallel to $E$, an incident light sees an index variation and the device operates as a pure polarization insensitive variable phase delay.
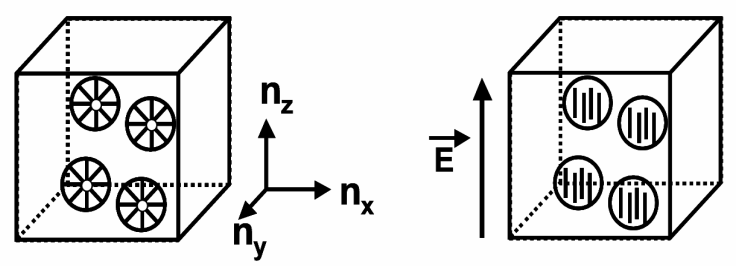

Fig. 1. Schematic representation of the nano-PDLC material behavior with and without an electric field.

Nano-PDLC exploits electro-optic properties of LC droplets. This approach is an interesting way to realize a tunable VCSEL because enabling more robust and compact solutions than with intra-cavity air gap modulation. However, nano-PDLC optical losses is a crucial parameter which implies important consequences on the lasing characteristics for the device. Losses, such as scattering by the LC droplets must be minimized. The LC droplets diameter is dependant of the UV power used during photo-polymerization-induced phase separation (PIPS) process (8) and have been estimated close to $100 \mathrm{~nm}$ in our case. For such a size, optical losses close to 15 $\mathrm{cm}^{-1}$ at $1.55 \mu \mathrm{m}$ have been measured for specific samples comprising a nano-PDLC layer sandwiched between two glass substrates. These optical losses are observed when LC droplets are randomly oriented without the application of an electric field.

Nano-PDLC based on small LC droplets are also interesting because exhibiting faster response times than bulk LC. The switching speed is related to the droplet size, and it improves as the droplet size is reduced. In contrast, electrooptic forces needed to switch LC droplets are consequently much higher. Thus, the driving voltage is also dependant of the droplet size, and can be expressed as (9):

$$
V_{d}=\frac{C}{\sqrt{D}}
$$

where $C$ is a constant and $D$ is the droplet size. A decrease in droplet size reduces the response time but requires higher voltages. But if an increase in droplet size decreases voltages, it reduces the speed and introduces scattering losses. This is the common trade-off for such a material.

\section{Device assembling}

Our tunable VCSEL comprises a nano-PDLC phase layer which is inserted between a half cavity and a dielectric distributed Bragg reflector (DBR). The half cavity is grown with a gas source molecular beam epitaxy in a single run, comprising InP/InGaAsP DBR and a periodic gain active region. The cavity has an optical length of three half-wave periods which contains three sets of seven lattice-matched
InGaAs/InGaAsP Quantum Wells (QW). Each multi-QWs (MQW) is located at an antinode of the standing wave for modal gain improvement. Each MQW is surrounded by $\mathrm{Q}_{1.18}$ layers and InP layers $\left(\mathrm{Q}_{1.18}\right.$ is a quaternary alloy $\mathrm{In}_{0.8} \mathrm{Ga}_{0.2} \mathrm{As}_{0.435} \mathrm{P}_{0.565}$ emitting at a $1.18 \mu \mathrm{m}$ wavelength). The $\mathrm{Q}_{1.18}$ layers thicknesses which surrounds each MQW are optimized to obtain an homogenous optical absorption of the pump power. The growth of the bottom DBR is achieved with InP and $\mathrm{Q}_{1.45}$ semiconductor materials which are used as low and high refractive index, respectively. The bottom DBR of the VCSEL is formed by 40 periods resulting in a theoretical reflectivity from cavity of $99 \%$ at $1.56 \mu \mathrm{m}$ wavelength. As illustrated in Fig. 2, the low refractive index between these two materials implies for our DBR a narrow spectral bandwidth. However, a calculated reflectance value higher than $98.5 \%$ can be hoped inside a spectral window greater than $30 \mathrm{~nm}$ centered around the $1.56 \mu \mathrm{m}$ resonant wavelength of the DBR. Thus, despite this low refractive index, the spectral window of the bottom DBR is in agreement with the tuning range targeted here.

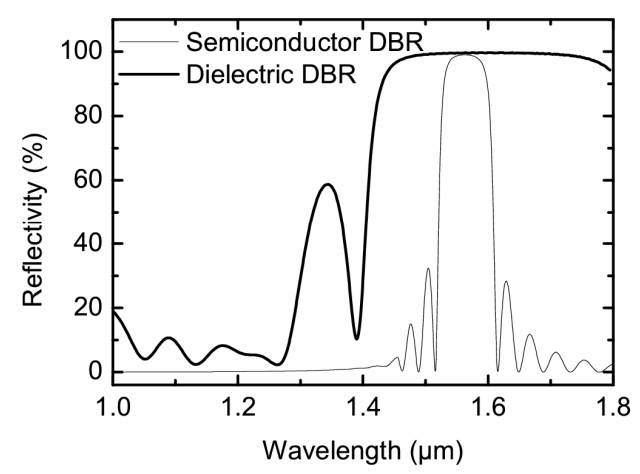

Fig. 2. Measured and simulated reflectivity obtained respectively for the dielectric and the semiconductor DBR..

The second mirror is deposited on a glass substrate with an electron-beam evaporation system. The large refractive index between the $\mathrm{TiO}_{2}$ and $\mathrm{SiO}_{2}$ dielectric materials $(\Delta n=$ 0.8 ) allows to reach a high reflectivity of $99.5 \%$ at $1.55 \mu \mathrm{m}$ with only 8.5 periods. Fig. 2 depicts the measured reflectivity of the DBR for a normal incidence. As it is illustrated, the spectral bandwidth is larger than the semiconductor DBR and layers thicknesses used are optimized to minimize the reflectivity around $6 \%$ at the pump wavelength of $1.064 \mu \mathrm{m}$.

Before the realization of this dielectric DBR, a thin semitransparent electrode (ITO: Indium Tin Oxide) layer is deposited on the glass substrate in order to apply the electric field on the phase layer. The second electrode consists in a $200 \mathrm{~nm}$ gold layer on the back of the N-doped InP substrate. The major part of the electric field is applied on the nanoPDLC phase layer and the dielectric DBR. Fig. 3 shows the final device where the half cavity and the front mirror are joined together thanks to micrometric spacers. These micrometric spacers allow to obtain an air gap between the half cavity and the dielectric DBR with an accuracy to within 
$2 \%$. This air-gap is filled with a mixture comprising nematic LC and UV-curable monomer. LC and the monomer were mixed at 30:70 wt\% ratio. In order to realize PIPS process, the mixture is UV cured through the dielectric Bragg mirror with strong UV illumination $\left(\lambda=365 \mathrm{~nm}\right.$ and $\left.P=350 \mathrm{~mW} / \mathrm{cm}^{2}\right)$ at RT. Consequently, LC droplets are formed with a size close to $100 \mathrm{~nm}$. Previously, the air-gap was measured to be $6 \mu \mathrm{m}$ corresponding to an optical length of $6 \lambda$. The tuning layer and active region thicknesses were chosen in order to reach high enough gain for laser emission and a relatively large tuning range.

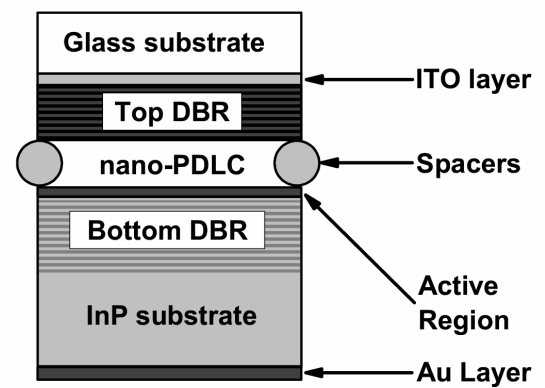

Fig. 3. Schematic cross-section of the tunable VCSEL.

\section{Results and discussion}

The photopumping experiment consists in focusing a laser beam with a microscope objective on the top mirror of the VCSEL with a spot area of $200 \mu \mathrm{m}^{2}$. The laser emission is collected back by the same objective and transmitted through a beam splitter. The signal is injected in a large core diameter optical fiber connected to a spectrum analyzer. The photopumping of the active region is realized by a pulsed Qswitched YAG laser at $1.064 \mu \mathrm{m}$ wavelength. This laser produces $1 \mathrm{~ns}-$ long pulses at a repetition rate of $6.6 \mathrm{kHz}$.

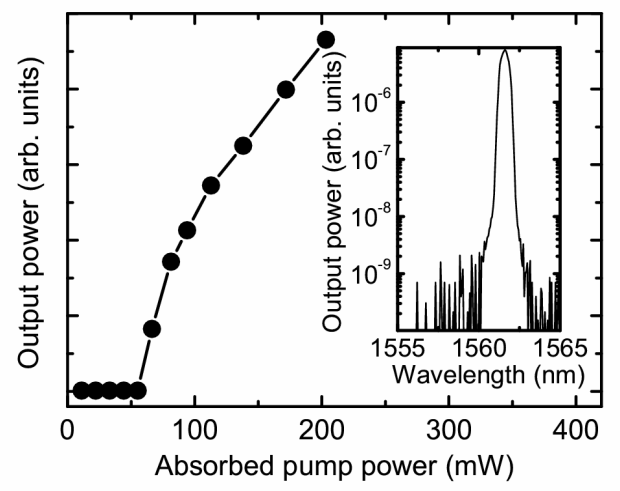

Fig. 4. Variation of the output power against absorbed pump power, for no applied voltage. The inset shows the laser spectrum for an absorbed pump power close to $100 \mathrm{~mW}$.

The output power emitted by the VCSEL versus the absorbed pump power at RT, for no applied voltage on the
nano-PDLC layer, is reported in Fig. 4. The fraction of the incident pump radiation absorbed in the active region has been estimated using a transfer matrix based model of the VCSEL structure. Thus, we assume that only $40 \%$ of the incident pump power radiation is actually absorbed in the active region. In these conditions, the incident pump power of 150 $\mathrm{mW}$ near the threshold corresponds to an absorbed pump power of about $60 \mathrm{~mW}$ (i.e. $30 \mathrm{~kW} / \mathrm{cm}^{2}$ ). This threshold value is high, but it can be explained by optical losses which are higher than the one expected. As mentioned above, the UV power used during PIPS process is a crucial parameter. The size of the LC droplets is dependent from the used UV power. In our case, a part of the incident UV power is absorbed by the $\mathrm{SiO}_{2} / \mathrm{TiO}_{2}$ dielectric Bragg mirror. Consequently, the size of the LC droplets is probably higher than $100 \mathrm{~nm}$ which implies optical losses higher than $15 \mathrm{~cm}^{-1}$. A laser spectrum, for an absorbed pump power close to $100 \mathrm{~mW}$, is reported in the inset of the Fig. 4. The peak power emitted by the VCSEL has been estimated close to $2 \mathrm{~mW}$ for such a pump power. The $1562 \mathrm{~nm}$ emission wavelength is in agreement with the maximum optical gain of the QWs and with the resonant wavelength of the bottom DBR.

Now, always with the same pump power, an external electric field have been applied on the nano-PDLC layer. Fig. 5 shows emission spectra under this constant optical pumping at different tuning voltage values. As the applied voltage between the top and bottom DBR is increased from 0 to 170 volts, the VCSEL wavelength is tuned from 1561.6 to 1551.8 nm.

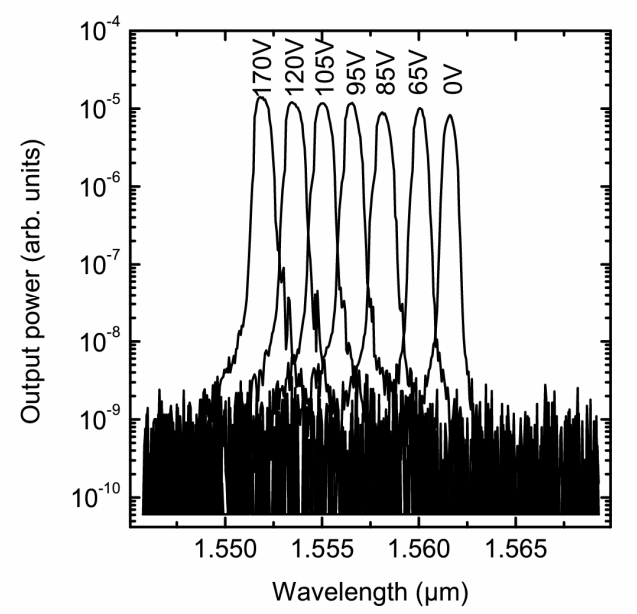

Fig. 5. Wavelength shift for different tuning voltage. Tuning voltage are indicated above each peak, in volts.

A tuning range of $9.8 \mathrm{~nm}$ is then demonstrated with an applied voltage close to $170 \mathrm{~V}$. As illustrated in Fig. 5, we notice the good single mode operation of the VCSEL with side-modes which are rejected at more than $20 \mathrm{~dB}$ for the whole tuning range.

The response time for our electro-optic material have been also characterized. The use of samples comprising a nano-PDLC layer sandwiched between two glass substrates 
covered with ITO layers have been studied. Observations have been made possible by measuring the intensity variation at 633 $\mathrm{nm}$ due to scattering which depends on the proportion of oriented LC droplets directors in our nano-PDLC layer. As we can see previously, the tuning response time is basically related to the size of the LC droplets. Typical switching responses of $100 \mu \mathrm{s}$ are obtained with our material choice and LC concentration. However, as seen on Fig. 5, for low and high voltage values, the wavelength shift is weak. Consequently, only a limited voltage range close to $90 \mathrm{~V}$ is enough to scan $90 \%$ of the wavelength range. Furthermore, the response time of the transition between the no applied voltage and the steady states is greatly reduced when applying a large voltage pulse during a short laps of time prior to apply a steady state voltage (10). These pre-orientation pulses can be used to speed up significantly the material without damages.

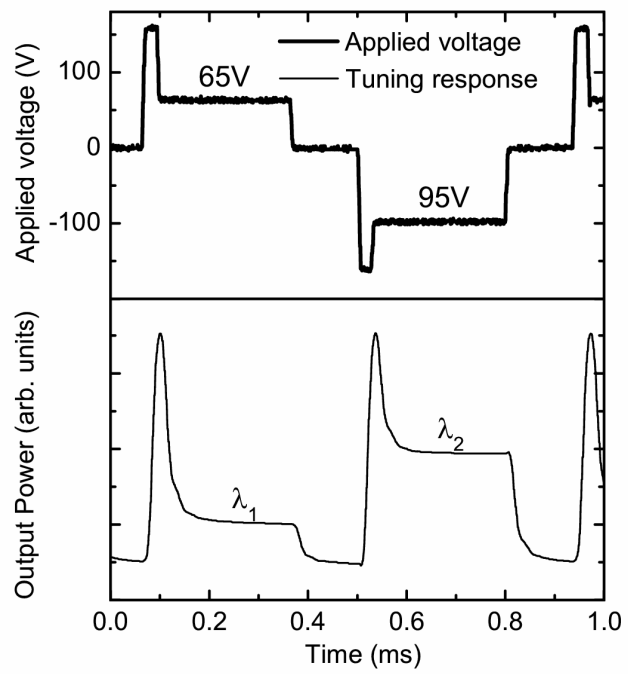

Fig. 6. Applied voltage and the measured response time of nano-PDLC material.

For instance, by using the addressing scheme presented in Fig. 6, it is possible to speed up the tuning time. Short prepulses of $25 \mu \mathrm{s}$ are used to switch the LC rapidly, before applying the voltage value corresponding to the chosen wavelength to be addressed and so far, for another wavelength value. As it can be seen in Fig. 6, wavelengths can be tuned in less than $30 \mu \mathrm{s}$, and the response time, for the considered voltage range, is not a function of the selected wavelength.

\section{Conclusion}

In conclusion, we demonstrated the first laser emission for a wavelength tunable $1.55 \mu \mathrm{m}$ VCSEL in which wavelength tuning is obtained via an electric field induced index modulation of a nano-PDLC layer. First characterizations have shown a tuning range equal to $10 \mathrm{~nm}$ under $170 \mathrm{~V}$ applied voltage. This preliminary demonstration has been achieved with an optical pumping. The device is easy to realize and do not need complex processing as in MEMS VCSEL. The preliminary switching times measurements shown that any wavelength can be selected in less than $30 \mu$ s. Finally, the high voltage value can be reduced by employing and introducing ITO electrodes with low optical losses inside the cavity, close to the nano-PDLC layer.

\section{Acknowledgment}

The authors wish to thank the Brittany region for supporting this project and the Kerdry company for manufacturing the dielectric Bragg mirrors.

\section{References}

1. C. J. Chang-Hasnain, "Tunable VCSEL”, IEEE J. Select. Topics in Quantum Electron., Vol. 6, pp. 978-987, 2000.

2. M. Maute, B. Kögel, G. Böhm, P. Meissner, and M.-C. Amann, "MEMS-tunable 1.55- $\mu \mathrm{m}$ VCSEL with extended tuning range incorporating a buried tunnel junction", IEEE Photon. Technol. Lett., Vol. 18, pp. 688-690, 2006.

3. D. Vakhshoori, P. Tayebati, C.C. Lu, M. Azimi, P. Wang, J.H. Zhou and E. Canoglu, "2 $\mathrm{mW}$ CW singlemode operation of a tunable 1550-nm VCSEL with 50-nm tuning range", Electron. Lett., Vol. 35, pp. 900-901, 1999.

4. F. Riemenschneider, M. Maute, H. Halbritter, G. Boehm, M.-C. Amann, and P. Meissner, "Continuously tunable long-Wavelength MEMS-VCSEL with over 40-nm tuning range", IEEE Photon. Technol. Lett., Vol. 16, pp. 22122214, 2004.

5. N. K. Dutta, and D. Vakhshoori, "Article comprising a tunable semiconductor laser”, US patent n`5301201, 1994.

6. S. Matsumoto, M. Houlbert, Takayoshi, and K.-ichi Kubodera, "Fine droplets of liquid crystals in a transparent polymer and their response to an electric field", Appl. Phys. Lett., Vol. 69, pp. 1044-1046, 1996.

7. P. J. W. Hands, A.K. Kirby, and G.D. Love, "Phase modulation with polymer-dispersed Liq. crystals" Proceedings of the SPIE, Vol 5894, August 2005.

8. L. Bouteiller, and P. Le Barny, "Polymer-dispersed liquid crystals: preparation, operation and application", Liq. Cryst., Vol. 21, pp. 157-74, 1996.

9. H. Ren, and S.-T. Wu, "Inhomogeneous nanoscale polymer-dispersed liquid crystals with gradient refractive index", Appl. Phys. Lett., Vol. 81, pp. 3537-3539, 2002.

10. S.T. Wu, and C.S Wu, "High speed nematic liquid crystal modulators", Mol. Cryst. Liq., Vol. 207, pp. 1-15, 1991. 